\title{
Direct Estimation of Inhomogeneous Markov Interval Models of Spike Trains
}

\author{
Daniel K. Wójcik \\ d.wojcik@nencki.gov.pl \\ Gabriela Mochol \\ g.mochol@nencki.gov.pl \\ Department of Neurophysiology, Nencki Institute of Experimental Biology, \\ 02-093 Warsaw, Poland
}

Wit Jakuczun

w.jakuczun@wlogsolutions.com

WLOG Solutions, Warsaw 02-389, Poland

Marek Wypych

m.wypych@nencki.gov.pl

Wioletta J. Waleszczyk

w.waleszczyk@nencki.gov.pl

Department of Neurophysiology, Nencki Institute of Experimental Biology,

02-093 Warsaw, Poland

A necessary ingredient for a quantitative theory of neural coding is appropriate "spike kinematics": a precise description of spike trains. While summarizing experiments by complete spike time collections is clearly inefficient and probably unnecessary, the most common probabilistic model used in neurophysiology, the inhomogeneous Poisson process, often seems too crude. Recently a more general model, the inhomogeneous Markov interval model (Berry \& Meister, 1998; Kass \& Ventura, 2001), was considered, which takes into account both the current experimental time and the time from the last spike. Several techniques were proposed to estimate the parameters of these models from data. Here we propose a direct method of estimation that is easy to implement, fast, and conceptually simple. The method is illustrated with an analysis of sample data from the cat's superior colliculus.

\section{Introduction}

The problem of information coding in sensory systems is one of the outstanding problems of neuroscience (Rieke, Warland, de Ruyter van Steveninck, \& Bialek, 1999; van Hemmen \& Sejnowski, 2006). It is particularly striking posing the variability of neural responses against the 
stability of our percepts. A natural approach to the problem of coding is through the theory of probability and information theory (Rieke et al., 1999). Even if the changes of membrane potential can be considered deterministic (Hodgkin \& Huxley, 1952) and if we neglect the synaptic noise (Faisal, Selen, \& Wolpert, 2008), the multitude of synaptic contacts nevertheless usually requires a statistical approach in the description of spike trains.

A natural framework for the description of spiking responses is provided by point process theory (Cox \& Isham, 1980; Daley \& Vere-Jones, 2003). It has been used in neuroscience for a long time (Perkel, Gerstein, \& Moore, 1967); however, two simplified approaches were most popular. Renewal processes, which could account for refractory properties of the membrane, were used to describe stationary processes. Nonstationarity has usually been described with inhomogeneous Poisson processes (Tuckwell, 1988). Only in the past two decades have other models started to come into use to describe nonstationary spike trains such as inhomogeneous renewal processes (Gerstner \& Kistler, 2002), inhomogeneous Markov interval (IMI) processes (Berry \& Meister, 1998; Kass \& Ventura, 2001), or time-rescaled renewal processes (Brown, Barbieri, Ventura, Kass, \& Frank, 2002).

To estimate models from data requires balancing the flexibility of the model versus the estimation precision given available data. Two-parameter processes, in particular, multiplicative IMI models, seem especially suitable in the neurophysiological context. They are flexible enough to account for the structure of the receptive fields and membrane properties yet simple enough to be reasonably estimated from the data that typically are available. Several approaches were proposed for estimating IMI models (Berry \& Meister, 1998; Kass \& Ventura, 2001; Truccolo, Eden, Fellows, Donoghue, \& Brown, 2005). We propose here a simple approach that we found conceptually intuitive, easy to implement, and efficient, even if not as general as the techniques based on the generalized additive models (Kass \& Ventura, 2001) or the generalized linear models (Truccolo et al., 2005). It is based on direct estimation of the part of the model describing history-dependent properties of spike generation under the assumption of a constant rate, followed by an estimation of the modulatory part describing the response properties. While in the long run one wants to move toward more complex models involving more covariates, for instance, including parametric models of receptive fields or assumed network topology (Truccolo et al., 2005; Pillow et al., 2008), the simple models such as those discussed here will keep their utility for describing experimental results, classification studies, and generating surrogate data.

In this note, we introduce the formal model to be estimated in the framework of general point processes in section 2. We then discuss our estimation method and a class of experiments where it is applicable in section 3 . The technique is illustrated with analysis of sample data from cat superior colliculus cells in section 4 . The results are summarized in section 5 . 


\section{Multiplicative Inhomogeneous Markov Interval Models}

Consider an experiment where a spike train is recorded from a neuron observed in time $t \in(0, T]$. We can describe the process locally in time with conditional intensity $\lambda$, also called the hazard function (Cox \& Isham, 1980; Johnson, 1996; Gerstner \& Kistler, 2002). It describes the probability density of generating a spike at time $t$ given the whole history of the process up to $t$, that is,

$$
\left\{\begin{array}{l}
\operatorname{Pr}\left[1 \text { event in }(t, t+\Delta t] \mid \text { spikes at } t_{1}, t_{2}, \ldots, s_{*}(t)\right] \\
\quad=\lambda\left(t ; t_{1}, t_{2}, \ldots, s_{*}(t)\right) \Delta t \\
\operatorname{Pr}\left[\text { more than } 1 \text { events in }(t, t+\Delta t] \mid \text { spikes at } t_{1}, t_{2}, \ldots, s_{*}(t)\right]=o(\Delta t) .
\end{array}\right.
$$

Here $t_{k}$ are times of consecutive spikes in a single realization of the process, $s_{*}(t)$ is the last spike time before $t$ in this realization, and $o(\Delta t)$ denotes terms of higher order than linear in $\Delta t$.

To estimate $\lambda$ from data, one is forced to assume memory of no more than the last few spikes. In the simplest case of intensity, depending on time only, $\lambda(t)$, this is the inhomogeneous Poisson process. If we relax the constraint and assume the dependence of the hazard function on the current time $t$ and time from the previous spike $\tau=t-s_{*}(t)$ we obtain the inhomogeneous Markov interval or inhomogeneous renewal process (Berry \& Meister, 1998; Kass \& Ventura, 2001; Gerstner \& Kistler, 2002). We further restrict ourselves to the multiplicative variant of the model of the form

$$
\lambda(t, \tau)=\lambda_{1}(t) \cdot \lambda_{2}(\tau)
$$

Berry and Meister (1998) proposed a simple method for estimating these factors from data. They assumed $\lambda_{2}(\tau)=0$ for $\tau$ less than the time of absolute refraction $t_{\mathrm{abs}}$ and $\lambda_{2}(\tau)=1$ for $\tau$ greater than the time of relative refraction, $t_{\text {rel }}$. The intermediate values were obtained from the probability distribution of all interspike intervals (ISI). Having thus obtained $\lambda_{2}(\tau)$, they used it to estimate $\lambda_{1}(t)$ from the mean firing rate. The apparent simplicity of this approach is hampered by several assumptions, which in general need not be satisfied. For example, the assumption of the special form of $\lambda_{2}(\tau)=0$ for $\tau<t_{\mathrm{abs}}$ and 1 for $\tau>t_{\text {rel }}$, while physiologically very natural, in general is unjustified. In fact, $\lambda_{2}(\tau)$ can even be unbounded (cf. the typical hazard functions; Tuckwell, 1988).

In response, more general alternative procedures were proposed by Kass and Ventura (2001) and Truccolo et al. (2005) based on, respectively, generalized additive models and generalized linear models. There, the idea is to span $\lambda_{1}(t)$ and $\lambda_{2}(\tau)$ on a spline basis with appropriately chosen knots and fit the spline parameters from data. These methods are much more universal but can be slow for large amounts of data due to substantial optimization needs. 
In our analysis of data from the cat's superior colliculus cells, we observed that the data did not satisfy the assumptions of Berry and Meister's (1998) procedure, and applying the approaches of Kass and Ventura (2001) and Truccolo et al. (2005) led to computationally intensive analysis. We found a simple variant of Berry and Meister's approach that proved easy to implement, efficient, and conceptually natural, but it can be applied to only a class of experiments including recordings of stationary activity. We discuss it in the next section.

\section{Estimation of IMI Process from Data}

Consider an experiment where a stimulus $s$ is presented $N$ times during intervals of length $T$. Assume also a control recording with no stimulus and stationary activity during time $T_{\text {stationary }}$. Thus, we have $N$ spike trains of duration $T$ and an additional spike train of duration $T_{\text {stationary }}$. We assume that the data are described by the multiplicative IMI model, equation 2.1. There is an undetermined constant in the two factors, and we set it by requiring $\lambda_{1}=1$ for stationary activity with no stimulus. An alternative natural normalization is to require $\lambda_{1}$ equal to the mean rate in this region. Thus, we can easily obtain $\lambda_{2}(\tau)$ using the standard approach for stationary renewal processes (Cox \& Lewis, 1966; Perkel et al., 1967). We estimate the probability density of interspike intervals $P(\tau)$ from the control recording of background activity. From $P(\tau)$, we obtain $\lambda_{2}$ as

$$
\lambda_{2}(\tau)=\frac{P(\tau)}{S(\tau)}=\frac{P(\tau)}{1-\int_{0}^{\tau} d \tau^{\prime} P\left(\tau^{\prime}\right)},
$$

where $S(\tau)=1-\int_{0}^{\tau} d \tau^{\prime} P\left(\tau^{\prime}\right)$ is the survival function. In practice, we used either nonparametric or parametric methods, which have different advantages and trade-offs (Hastie, Tibshirani, \& Friedman, 2001). In the nonparametric approach, we used gaussian kernel smoothing with optimal or scaled kernel width and with positive support (Bowman \& Azzalini, 1997). ${ }^{1}$ In the parametric variant, we fitted gamma distribution. The analysis was done using Matlab.

Having obtained the factor describing refractory properties of the membrane, $\lambda_{2}(\tau)$, we can evaluate the modulatory factor $\lambda_{1}(t)$ describing the cell's response properties. We divide the time of experiment, $(0, T]$, into bins of length $\Delta t$ short enough that there would be at most one spike per bin. The probability of generating a spike in trial $j$ in bin $k$ is approximately $p_{k}^{j}=\lambda_{1}\left(t_{k}\right) \lambda_{2}\left(\tau_{k}^{j}\right) \Delta t$, where $t_{k}=(k-1 / 2) \Delta t, \tau_{k}^{j}=t_{k}-s_{*}^{j}\left(t_{k}\right)$, and $s_{*}^{j}(t)$ is the

\footnotetext{
${ }^{1}$ We first used ksdensity function with gaussian kernel to evaluate optimal window width $u$. Then we used it to calculate the cumulative density function and probability density function with window width $0.5 u, u$, and $1.5 u$.
} 
time of the last spike before $t$ on $j$ th trial. If no history of spike train is known before $t=0$, we assume $\tau=t$ until the first spike, $t_{1}^{j}$. The possible error introduced by inexact timing was usually negligible for typical spike statistics. Over $N$ repetitions, the mean probability of observing a spike in bin $k$ is

$$
p_{k}=\left\langle p_{k}^{j}\right\rangle_{j}=\frac{1}{N} \sum_{j} p_{k}^{j}=\lambda_{1}\left(t_{k}\right) \Delta t \frac{1}{N} \sum_{j=1}^{N} \lambda_{2}\left(\tau_{k}^{j}\right) .
$$

But $p_{k}$ is essentially time-dependent rate $p_{k}=r_{k} \Delta t=N_{k} / N$, where $N_{k}$ is the number of trials on which we observed a spike in bin $k$ and the rate $r_{k}=(1 / \Delta t)\left(N_{k} / N\right)$. Since we already know $\lambda_{2}$, we obtain an estimate for $\lambda_{1}$ as

$$
\lambda_{1}\left(t_{k}\right)=\frac{N r_{k}}{\sum_{j=1}^{N} \lambda_{2}\left(\tau_{k}^{j}\right)} .
$$

In practice, we obtained the rate by either smoothing the poststimulus time histogram with a gaussian kernel or by spreading each individual spike with a gaussian kernel with $\sigma=5$ or $10 \mathrm{~ms}$ and averaging the sum (Nawrot, Aertsen, \& Rotter, 1999). Usually the results were equivalent. Since the mean $\lambda_{2}(\tau)$ was rather variable, to stabilize the resulting $\lambda_{1}$, we also smoothed the time-dependent function $\sum_{j=1}^{N} \lambda_{2}\left(\tau_{k}^{j}\right)$ with a Savitzky-Golay filter of order 3 and width $31 \mathrm{~ms}$.

The whole scheme easily generalizes to a situation where a set of stimuli $s_{i}, i=1, \ldots, K$ is presented repeatedly, $n_{i}$ times each. We discuss a simple example of experimental data analysis in such a case in the next section.

\section{Results}

To illustrate our estimation method, we used the data of the single unit recording from the cat's superior colliculus. Conventional experimental methods for animal preparation and extracellular single unit recording were used (Waleszczyk, Wang, Burke, \& Dreher, 1999). In the experiment, spike trains of single neurons were recorded during movements of a bar of light on a screen with a fixed velocity along one axis of the receptive field and waiting periods, when the stimulus was held outside the receptive field for 1 second between the sweeps in both directions. Single cell data consisted of multiple recordings of responses to stimuli of different velocities. Velocities ranged from 2 to 1000 degrees per second. For short sweeps with high velocities, we could see the response extending to the first part of the waiting period. However, it was never noticeable during the last $0.5 \mathrm{~s}$ of the waiting period. We pooled all the intervals from all such periods following sweeps into a single collection of ISIs. This procedure was equivalent to the analysis of 
a single long recording of background activity advocated in the previous section.

The probability distribution of ISIs sampled by this collection was used to estimate $\lambda_{2}(\tau)$. Thus, we assumed $\lambda_{1}(t)=1$ in the second half of each waiting period. We used both parametric and nonparametric methods for estimating $\lambda_{2}(\tau)$, as mentioned in the previous section.

Given $\lambda_{2}$, we analyzed responses to every stimulus separately using the procedure detailed in the previous section. Figure 1 shows example results of such analysis. These data are from a single cell and a single stimulus $(v=1000 \mathrm{deg} / \mathrm{s}$ moving left to right). Figure 1A shows the distribution of all the ISIs from the background activity (empirical data; bar plot), the nonparametric estimate (solid line), and the parametric fit (gamma distribution; dashed line) of the probability distribution $P(\tau)$. The main plot in log-normal coordinates shows the differences in the tails, and the inset in normal-log coordinates emphasizes the differences for small intervals.

Figure $1 \mathrm{~B}$ shows the estimate of $\lambda_{2}(\tau)$ obtained from the data in Figure $1 \mathrm{~A}$. There is a striking difference between the nonparametric and parametric estimates for $\tau>0.1 \mathrm{~s}$, as the hazard function for gamma distribution is monotonic while the nonparametric estimate is more flexible. To test the stability of estimates, we calculated $\lambda_{2}$ on different parts of data. We separated waiting periods following stimuli moving left to right from those following stimuli moving right to left. We also analyzed separately the intervals from the first half of the recordings and those from the second half of the recordings. In all cases for these data, the obtained results were quantitatively very similar to the result obtained from all of the data (not shown).

In Figure 1C we show $\lambda_{1}(t)$ estimated from data as described in section 3 using a nonparametric (solid line) and a parametric approach (dashed line). To compare these results with the inhomogeneous Poisson model (dashdotted curve), all have been normalized so that their mean value during the waiting period is equal to 1 . There is an enhancement of the response profile in both IMI models as compared to the Poisson model, particularly strong for the nonparametric model, that corroborates the previous findings well (Berry \& Meister, 1998; Kass \& Ventura, 2001).

Figure 1D compares the quality of different models using the Kolmogorov-Smirnov (K-S) plots (Brown et al., 2002). Each curve was obtained by appropriate rescaling of spike times (Brown et al., 2002) using conditional intensity estimated with the inhomogeneous Poisson model (dash-dotted curve), the parametric IMI model (dashed curve), or the nonparametric IMI model (thick solid line). A perfect model of data corresponds to the diagonal (thin solid line), and two parallel thin dashed lines demark the $95 \%$ confidence bounds. We interpret the distance from the diagonal as a measure of the quality of the model.

Clearly the inhomogeneous Poisson model is describing the data rather poorly. The strong deviation observed for short intervals is expected, as 
(A)

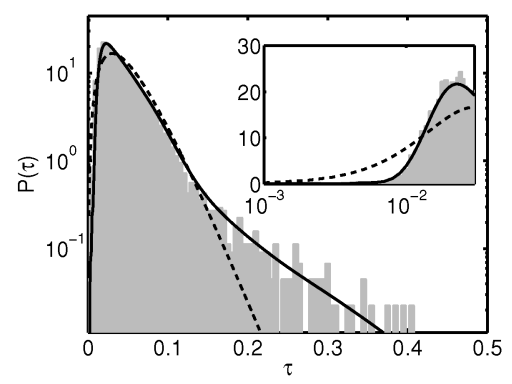

(C)

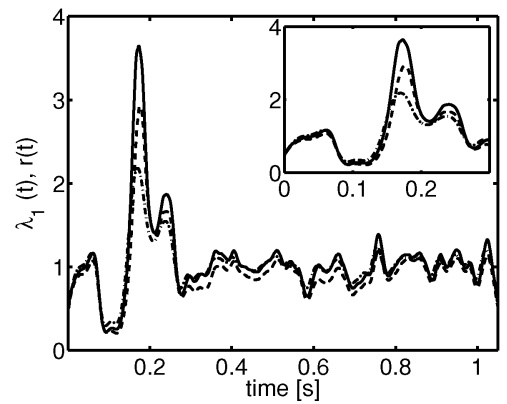

(B)

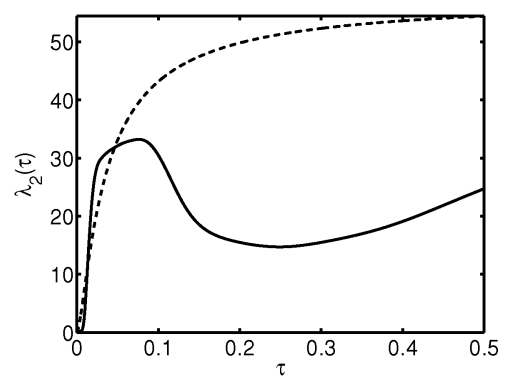

(D)

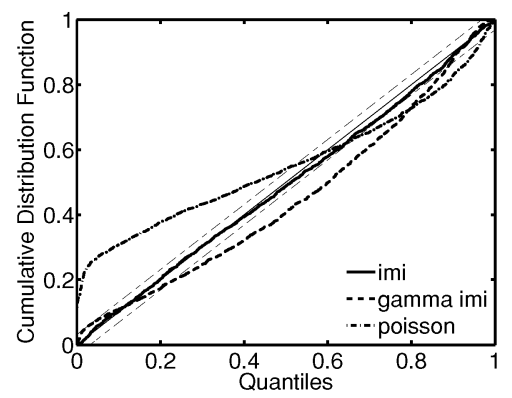

Figure 1: Estimation of IMI models from sample data: a single cell and a single stimulus ( $v=1000 \mathrm{deg} / \mathrm{s}$ moving left to right). (A) Distribution of all the ISIs from the background activity (bar plot), the nonparametric estimate (solid line), and the parametric fit (gamma distribution; dashed line). The main plot in log-normal coordinates shows the difference in the tails. The inset in normallog coordinates emphasizes the differences for small intervals. (B) The estimate of $\lambda_{2}(\tau)$ obtained from the distribution shown in $A$. (C) The modulatory factor $\lambda_{1}(t)$ estimated from data as described in section 3 using a nonparametric (solid line) and a parametric approach (dashed line). To compare these results with the inhomogeneous Poisson model (dash-dotted curve), they have been normalized so that their mean value during the waiting period is equal to 1 . (D) The quality of different models using the Kolmogorov-Smirnov plots. Each curve was obtained by appropriate rescaling of spike times using conditional intensity estimated with an inhomogeneous Poisson model (dash-dotted curve), parametric IMI model (dashed curve), or nonparametric IMI model (thick solid line). A perfect model of data corresponds to the diagonal (thin solid line). Two parallel thin dashed lines demark a 95\% confidence bound. 
this model does not take into account the refractory properties of the membrane. The gamma IMI gives a much better description of the data, with a good description of short intervals (within 95\% confidence bounds). The relatively poor description of the distribution of longer intervals in the gamma IMI model is a consequence of the stiffness of the parametric model (cf. Figure 1B). The best description of these data is provided by the nonparametric IMI model: the K-S curve obtained with this model lies entirely within the $95 \%$ confidence bound.

\section{Summary}

The common model of spike trains, the inhomogeneous Poisson process, is very useful in its simplicity and often adequate for describing experimental data, especially for relatively low firing rates. To account for the membrane mechanisms such as refraction, one must go beyond the Poisson processes, and the inhomogeneous Markov interval models seem good candidates for modeling spike train data. We proposed a direct method useful in describing experimental recordings where, apart from responses to repeated stimuli, background activity was also recorded. Our proposition was to use the stationary data from the background activity to estimate the factor of intensity describing the membrane properties, $\lambda_{2}(\tau)$. An estimate of $\lambda_{2}(\tau)$ can then be used to extract the modulatory factor, taking into account the response properties of the cell, $\lambda_{1}(t)$. We have demonstrated a practical use of our method on data from an example cell from the cat's superior colliculus and showed with K-S plots the superiority of the IMI model over the inhomogeneous Poisson model for these data. A complete physiological analysis of the full dataset from these experiments is in preparation. We believe that the simplicity of our estimation method will make it a viable alternative to other approaches wherever it can be applied.

\section{Acknowledgments}

We have benefited from discussions with Szymon Łęski. This research is funded by the Polish Ministry of Science and Higher Education, research grants N401 146 31/3239 and 46/N-COST/2007/0.

\section{References}

Berry, M. J., \& Meister, M. (1998). Refractoriness and neural precision. J. Neurosci., 18(6), 2200-2211.

Bowman, A., \& Azzalini, A. (1997). Applied smoothing techniques for data analysis. New York: Oxford University Press.

Brown, E. N., Barbieri, R., Ventura, V., Kass, R. E., \& Frank, L. M. (2002). The timerescaling theorem and its application to neural spike train data analysis. Neural Comput., 14(2), 325-346. 
Cox, D. R., \& Isham, V. (1980). Point processes. London: Chapman and Hall.

Cox, D. R., \& Lewis, P. A. W. (1966). The statistical analysis of series of events. London: Chapman and Hall.

Daley, D. J., \& Vere-Jones, D. (2003). An introduction to the theory of point processes. Berlin: Springer.

Faisal, A. A., Selen, L. P. J., \& Wolpert, D. M. (2008). Noise in the nervous system. Nat. Rev. Neurosci. 9(4), 292-303.

Gerstner, W., \& Kistler, W. M. (2002). Spiking neuron models. Cambridge: Cambridge University Press.

Hastie, T., Tibshirani, R., \& Friedman, J. (2001). The elements of statistical learning. Berlin: Springer.

Hodgkin, A., \& Huxley, A. (1952). A quantitative description of membrane current and its application to conduction and excitation in nerve. Journal of Physiology, $117,500-544$.

Johnson, D. H. (1996). Point process models of single-neuron discharges. J. Comput. Neurosci., 3(4), 275-299.

Kass, R. E., \& Ventura, V. (2001). A spike-train probability model. Neural Comput., 13(8), 1713-1720.

Nawrot, M., Aertsen, A., \& Rotter, S. (1999). Single-trial estimation of neuronal firing rates: From single-neuron spike trains to population activity. J. Neurosci. Methods, 94(1), 81-92.

Perkel, D. H., Gerstein, G. L., \& Moore, G. P. (1967). Neuronal spike trains and stochastic point processes. I. The single spike train. Biophys J., 7(4), 391-418.

Pillow, J. W., Shlens, J., Paninski, L., Sher, A., Litke, A. M., Chichilnisky, et al. (2008). Spatio-temporal correlations and visual signalling in a complete neuronal population. Nature, 454(7207), 995-999.

Rieke, F., Warland, D., de Ruyter van Steveninck, R., \& Bialek, W. (1999). Spikes: Exploring the neural code. Cambridge, MA: MIT Press.

Truccolo, W., Eden, U. T., Fellows, M. R., Donoghue, J. P., \& Brown, E. N. (2005). A point process framework for relating neural spiking activity to spiking history, neural ensemble, and extrinsic covariate effects. Journal of Neurophysiology, 93(2), 1074-1089.

Tuckwell, H. C. (1988). Introduction to theoretical neurobiology. Cambridge: Cambridge University Press.

van Hemmen, J. L., \& Sejnowski, T. J. (Eds.). (2006). 23 problems in systems neuroscience. New York: Oxford University Press.

Waleszczyk, W. J., Wang, C., Burke, W., \& Dreher, B. (1999). Velocity response profiles of collicular neurons: Parallel and convergent visual information channels. Neuroscience, 93(3), 1063-1076.

Received July 25, 2008; accepted January 26, 2009. 